\title{
A GC-MS Based Metabolic Profiling of Probiotic Lactic Acid Bacteria Isolated from Traditional Food Products
}

\author{
Aditya Chaudhary $^{1} \mathbb{D}$, Khushbu Verma ${ }^{1 *} \mathbb{D}$ and Baljeet Singh Saharan ${ }^{2}$ \\ ${ }^{1}$ Faculty of Agriculture and Veterinary Science, Jayoti Vidyapeeth Women's University, Jaipur, India. ${ }^{2}$ Department \\ of Microbiology, CCS Haryana Agricultural University, Hisar, Haryana, India.
}

\begin{abstract}
A GC-MS based metabolic profiling was carried out to study metabolic differences of lactic acid bacteria isolated from different food sources. Metabolic fingerprinting is a non-targeted procedure where all detectable peaks are considered to establish sample classification. A total of $\mathbf{4 0}$ compounds were identified as major metabolites contributing to the difference among five different probiotic lactic acid bacteria. Some of the metabolites identified in this study have been reported as a defrosting agent, antioxidant, flavour agent, antimicrobial, natural food additive, anti-inflammatory, anti-sleep disorder agent and anti-cancer agents. These results suggest that GC-MS based metabolomic analysis is a useful tool to facilitate future investigations into the characterization of probiotic lactic acid bacteria.
\end{abstract}

Keywords: Lactic acid bacteria, metabolic profiling, GC-MS, traditional food products, probiotics

*Correspondence: vermakhushbu25@gmail.com

(Received: December 01, 2019; Accepted: February 20, 2020)

Citation: Aditya Chaudhary, Khushbu Verma, Baljeet Singh Saharan, A GC-MS Based Metabolic Profiling of Probiotic Lactic Acid Bacteria Isolated from Traditional Food Products, J. Pure Appl. Microbiol., 2020; 14(1):657-672. https://doi.org/10.22207/ JPAM.14.1.68

(C) The Author(s) 2020. Open Access. This article is distributed under the terms of the Creative Commons Attribution 4.0 International License which permits unrestricted use, sharing, distribution, and reproduction in any medium, provided you give appropriate credit to the original author(s) and the source, provide a link to the Creative Commons license, and indicate if changes were made. 


\section{INTRODUCTION}

Lactobacillus and Pediococcus is a grampositive facultative anaerobic bacterium found widely in fermented food products and can be investigated for the metabolites present in them by GC-MS based metabolic profiling.

Metabolic fingerprinting is explained as the semi-quantitative investigation of extracellular (exo-metabolome) and intracellular (endometabolome) metabolites, respectively (VillasBoas et al., 2005). These metabolic profiles GC-MS has consistently been the most favoured analytical technique for the analysis of metabolites present in distinct biological samples. Gas chromatography and mass spectrophotometry (GC-MS) present the high chromatographic resolution ascribed to the high sensitivity and specificity of mass spectrophotometry (Villas-Boas et al., 2005). In comparison with other techniques, GC-MS can produce a comparably high reproducibility, high resolution, high-quality sensitivity, and good- throughput analysis, which can be used for analyzing the metabolic products, inclusive of carbohydrates, fatty acids, organic acids, and amino acids (Park et al., 2016). Derivatization is necessary before the investigation by GC-MS as most of the metabolites are non-volatile (Schummer et al., 2009). Nowadays, most adaptable derivatization technique is silylation, which has the ability to derivatize compounds having polar functional groups by mixing TMS (Trimethylsilyl) reagent to develop TMS Compounds (Nordström A, 2004). However, for the study of different biological metabolic fingerprinting, N, O-bis (trimethylsilyl) trifluoroacetamide with $1 \%$ trimethylchlorosilane is generally used as silylation reagent and derivatization method with GC-MS (Alreshidi et al., 2015; Li et al., 2013). For the study of metabolites in the microbial sample, derivatization based GC-MS technique can be applied to identify the intracellular as well as extracellular metabolites of Lactobacillus and Pediococcus species.

Liquid injection GC-MS provides an inexpensive and simple option for the analysis of metabolites produced by probiotic lactic acid bacteria. As the concentration of metabolites varies widely in different probiotic lactic acid bacteria, it is required to develop the analytical plan that would permit concurrent quantification of them in a single run utilizing comparably a small amount of sample along with minimum sample preparation. GC-MS has high sensitivity and hence can be used for the investigation of less common samples that might only be available in minute quantity. In this study, direct-injection GC-MS methodology was used for the profiling of different metabolites, which has the high specificity of mass spectrometry along with the high reproducibility and high resolution of gas chromatography.

The results of this study provide reference data for interpreting the differences in the metabolite profiles of different probiotic lactic acid bacteria isolated from different food sources.

\section{MATERIALS AND METHODS Collection of food samples}

Food items viz. Dosa batter, jalebi batter, maida dough, sauerkraut and soymilk were used in this experiment. Dosa batter, Jalebi batter, Soymilk were collected from a local market in New Delhi, India. Maida dough and Sauerkraut were prepared at home. The food samples were taken in a sterilized bag and stored at $-4^{\circ}$ until use.

\section{Strain isolation}

$1 \mathrm{~g}$ or $1 \mathrm{ml}$ of food sample were added into $9 \mathrm{ml}$ of $0.85 \%(\mathrm{w} / \mathrm{v})$ normal saline. After homogenization, serial dilutions were prepared upto $10^{-9}$ with $0.85 \%(\mathrm{w} / \mathrm{v})$ normal saline and $0.1 \mathrm{ml}$ decimal of appropriate dilutions were plated onto de Man, Rogosa, Sharpe (MRS) agar medium (Himedia, India) (de Man et al., 1960). The agar plates were incubated at $35^{\circ}$ for $24 \mathrm{~h}$ under anaerobiosis. Morphologically different colonies were picked and re-streaked onto MRS agar plates up to purity. Glycerol stocks of strains were preserved at $-20^{\circ}$.

\section{Probiotic Lactic Acid Bacteria}

L. plantarum DB-2, L. fermentum J-1, $P$. acidilactici $\mathrm{M}-3$, L. plantarum SK-3 and $P$. pentosaceus SM-2 were isolated from Dosa batter, Jalebi batter, Maida dough, Sauerkraut, and Soymilk and were identified in own previous studies with NCBI accession no. MK246169, MK353735, MK461878, MK246167 and MK461882 respectively (Chaudhary and Saharan, 2019) (Table 1). Probiotic attributes such as acid tolerance (Liong and Shah, 2005), bile tolerance (Walker and Gilliland, 1993), antibiotic susceptibility (Thirabunyanon et al., 2009), hemolytic activity (Harrigan, 1998), gelatinase activity (Harrigan, 
1990), autoaggregation (Del Re et al., 2000), co-aggregation studies (Del Re et al., 2000), hydrophobicity (Rosenberg et al., 1980), bacteriocin production (Papagianni and Anastasiadou, 2009), lactic acid and hydrogen peroxide production (AOAC, 1995), exopolysaccharide production (Mora et al., 2002) were studied on all the five isolates. Bacterial isolates were identified at the genomic level by using $16 \mathrm{~S}$ rRNA gene technique (Hashem et al., 2010; Bollag and Edelstein, 1991). Metabolic fingerprinting

Metabolites were extracted by following the method given by Coucheney et al. (2008) with minor modifications.

\section{Sampling for metabolic fingerprinting}

Microbial suspensions $(20 \mathrm{ml})$ of isolate (grown in MRS broth for $24 \mathrm{~h}$ at $35^{\circ} \mathrm{C}$ ) were disrupted using pulsed, high-frequency sound waves $(>20 \mathrm{kHz}$ ) for five cycles $(30 \mathrm{sec}$. run; one min break) to extract intracellular metabolites. Suspensions procured after sonication were centrifuged for $10 \mathrm{~min}$ at $10,000 \mathrm{rpm}$ in order to segregate the extra as well as intracellular metabolites from the cells.

\section{Extraction for metabolic fingerprinting}

The supernatant was removed and the metabolites were extracted with a Methanol: Water: Chloroform mixture (2: $0.8: 1): 2.5 \mathrm{ml}$ of cold chloroform and $5 \mathrm{ml}$ of cold methanol $\left(-20^{\circ} \mathrm{C}\right)$ and the phases were allowed to separate. Metabolic fingerprints were assessed in the aqueous phase after freeze-drying while in the organic phase, the dried chemical extracts obtained after complete evaporation of the solvent was used.

Generation of metabolic fingerprints and data processing

Derivatization of dried samples

The derivatization method given by Mastrangelo et al. (2015) and Park et al. (2019) was used in this study for the evaluation of metabolic fingerprinting. The freeze-dried samples and dried chemical extracts were methoxymated using methoxyamine in pyridine solution and trimethylsilylated by BSTFA (N,O-Bis(trimethylsilyl) trifluoroacetamide) with $1 \%$ chlorotrimethylsilane (TMCS).

\section{GC-MS analysis}

Derivatives from Lactobacillus and Pediococcus strains were analysed using Thermo Trace $1300 \mathrm{GC}$ coupled with Thermo TSQ 800 Triple Quadrupole MS (Pogacic et al., 2015). Sixmicrolitre aliquots were injected in splitless mode. The samples were warmed to $65^{\circ} \mathrm{C}$ for $15 \mathrm{~min}$ and the metabolites were extracted before being adsorbed on the trap at $35^{\circ} \mathrm{C}$. The trap was heated at $250^{\circ} \mathrm{C}$ for $0.1 \mathrm{~min}$, leading to desorption of the metabolites. Metabolites were then separated on TG $5 \mathrm{MS}$ column $(30 \mathrm{~m} \times 0.25 \mathrm{~mm}, 0.25 \mu \mathrm{m}$ thickness) with column makeup of $5 \%$ diphenyl; $95 \%$ dimethyl polysiloxane. The temperature of the oven was initially $50^{\circ} \mathrm{C}$, maintained for $3 \mathrm{~min}$. The temperature was increased at $15^{\circ} \mathrm{C} / \mathrm{min}$ to $220^{\circ} \mathrm{C}$. The mass spectrometer was operated in the scan mode within a mass range of $m / z 30$ to 700 . The quadruple mass spectrophotometry parameters were set to the conditions: ion source temperature of $230^{\circ} \mathrm{C}$, split flow of $40 \mathrm{ml} / \mathrm{min}$, carrier flow of $1.5 \mathrm{ml} / \mathrm{min}$, injector temperature and MS transfer line temperature of $250^{\circ} \mathrm{C}$. Ionization was done by electronic impact at $70 \mathrm{eV}$. All samples were analysed in the same GC-MS and injected in a randomized order over the GC-MS run. Blank samples (boiled deionized water) were injected after every sample to verify the instrumental carryover.

Data pre-processing and data analysis

The GC-MS raw data files were converted to netCDF format. Further, the raw

Table 1. Genotyping of screened lactic acid bacterial strains

\begin{tabular}{lcccc}
\hline $\begin{array}{l}\text { Name of } \\
\text { isolate }\end{array}$ & Source & $\begin{array}{c}\text { Identity } \\
(\%)\end{array}$ & $\begin{array}{c}\text { 16S rRNA } \\
\text { identification }\end{array}$ & $\begin{array}{c}\text { Accession } \\
\text { number }\end{array}$ \\
\hline DB-2 & Dosa batter & 99 & Lactobacillus plantarum & MK246169 \\
J-1 & Jalebi batter & 99 & $\begin{array}{c}\text { Lactobacillus fermentum } \\
\text { Pediococcus acidilactici }\end{array}$ & MK353735 \\
M-3 & Maida dough & 97 & MK461878 \\
SK-3 & Sauerkraut & 100 & Lactobacillus plantarum & MK246167 \\
SM-2 & Soymilk & 99 & Pediococcus pentosaceus & MK461882 \\
\hline Journal of Pure and Applied Microbiology & & 659 & www.microbiologyjournal.org
\end{tabular}


Table 2. Metabolic profile of biocomponents identified in solvent extract of probiotic lactic acid bacteria isolated by GC-MS

\begin{tabular}{|c|c|c|c|c|c|c|}
\hline S.No & Compound & $\begin{array}{c}\text { Five } \\
\text { isolates }\end{array}$ & $\begin{array}{l}\text { Four } \\
\text { isolates }\end{array}$ & $\begin{array}{l}\text { Three } \\
\text { isolates }\end{array}$ & $\begin{array}{c}\text { Two } \\
\text { isolates }\end{array}$ & $\begin{array}{l}\text { One } \\
\text { isolate }\end{array}$ \\
\hline 1 & $\begin{array}{l}\text { Acetic acid, anhydride } \\
\text { with formic acid }\end{array}$ & & & & & - \\
\hline 2 & 4-amino-1-butanol & & & & & घ \\
\hline 3 & Benzaldehyde,2,4,dimethyl & 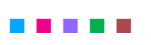 & & & & \\
\hline 4 & Benzoic acid & घa $=\square$ & & & & \\
\hline 5 & Decane & $\square \square \square \square$ & & & & \\
\hline 6 & DL-2,3-Butanediol & & & & 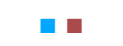 & \\
\hline 7 & Dodecane & घa $= \pm$ & & & & \\
\hline 8 & Dodecanoic acid & $\square \square \square \square$ & & & & \\
\hline 9 & Eicosanoic acid & & & $\square=\square$ & & \\
\hline 10 & Ethanamine, 2-propoxy- & & & & m & \\
\hline 11 & Ethanol, 2-(2-propenyloxy) & & & & 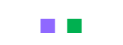 & \\
\hline 12 & 2-Ethoxyethylamine & & & & & घ \\
\hline 13 & Ethylamine & & & & & - \\
\hline 14 & Formamide & & & & & a \\
\hline 15 & Geranyl isovalerate & 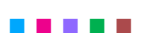 & & & & \\
\hline 16 & Hexadecane & 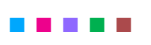 & & & & \\
\hline 17 & Hexadecane,2,6,11,15 tetramethyl & 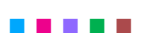 & & & & \\
\hline 18 & n-hexadecanoic acid & $\square \square \square \square$ & & & & \\
\hline 19 & 2-Hydrazino ethanol & & & $\mathbf{\square} \mathbf{\square}$ & & \\
\hline 20 & Hydroperoxide, heptyl & & & & & ! \\
\hline 21 & Hydroperoxide, 1-methylhexyl & & & & & - \\
\hline 22 & Hydroperoxide, pentyl & & & & $\square \mathbf{m}$ & \\
\hline 23 & Isopropyl Alcohol & 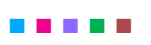 & & & & \\
\hline 24 & Isopropyl myristate & & $\square=\square$ & & & \\
\hline 25 & L-Lactic acid & 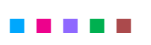 & & & & \\
\hline 26 & Methane, nitroso- & & 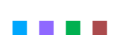 & & & \\
\hline 27 & Methyltartronic acid & & & & $\mathbf{\square}$ & \\
\hline 28 & 9 Octadecenamide, (Z) & $\square \square \square \square$ & & & & \\
\hline 29 & 4-Penten-2-ol & & & & & - \\
\hline 30 & Phenol,2,4,bis (1,1 dimethylethyl) & $\square \square \square \square$ & & & & \\
\hline 31 & Propanoic acid, 2-hydroxy-, methyleste & & & & & a \\
\hline 32 & 2-Propanol,1-Hydrazino & 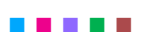 & & & & \\
\hline 33 & Propylene glycol & & $\square=\square$ & & & \\
\hline 34 & $\begin{array}{l}\text { Pyrrolo [ } 1,2 \text { a ] pyrazine 1,4 dione, } \\
\text { hexahydro } 3 \text { ( } 2 \text { methylpropyl) }\end{array}$ & 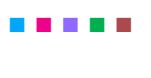 & & & & \\
\hline 35 & $\mathrm{R}-(-)-1,2$ propanediol & & $\mathbf{\square} \mathbf{m} \mathbf{m}$ & & & \\
\hline 36 & Squalene & & & & घ & \\
\hline 37 & Tetracosane & & घ घ घ & & & \\
\hline 38 & Tetradecane & 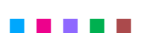 & & & & \\
\hline 39 & Tetradecanoic acid & & घ घ & & & \\
\hline 40 & Undecane & m a a a & & & & \\
\hline
\end{tabular}

- represents Lactobacillus plantarum DB-2

- represents Lactobacillus fermentum J-1

- represents Pediococcus acidilactici M-3

- represents Lactobacillus plantarum SK-3

- represents Pediococcus pentosaceus SM-2 
data were processed to time- and mass- aligned chromatographic peak areas with the XCalibur 2.2SP1 with Foundation 2.0SP1. Metabolites were identified by comparison of mass spectra and retention times with those of authentic standards from NIST 2.0 Mass Spectral Library (Mastrangelo et al., 2015).

\section{RESULTS AND DISCUSSION Metabolic fingerprinting}

The exo- and endo-metabolites of probiotic isolates in culture supernatant provides a window for explaining the comprehensive nature of metabolites. The metabolite profiling provides information about the nutritional as well as of toxic effects, if any of metabolites on the interactive effects of the dietary components on the health of the gut of the host.

In total, 40 metabolites were detected from the culture supernatants of these five isolates. Out of 40 compounds obtained in the solvent phase, only 17 compounds viz. Isopropyl alcohol; L-lactic acid; 2-Propanol, 1-hydrazino; Decane; Benzaldehyde, 2,4, dimethyl; Dodecane; Tetradecane; Phenol, 2,4 bis (1,1 dimethylethyl); Hexadecane, 2,6,11,15 tetramethyl; Hexadecane; Geranyl isovalerate; Pyrrolo [1,2a] pyrazine 1,4 dione, hexahydro 3 (2 methylpropyl); $\mathrm{n}$-Hexadeconic acid; 9 Octadecenamide, (Z); Benzoic acid; Undecane; Dodecanoic acid were common to all the tested isolates as described in Table 2. Out of 17 compounds, Isopropyl alcohol; Dodecane, Hexadecane, Tetradecane and Pyrrolo [1,2a] pyrazine 1,4 dione, hexahydro 3 (2 methylpropyl); Benzaldehyde, 2,4, dimethyl and Geranyl isovalerate; Phenol, 2,4 bis $(1,1$ dimethylethyl); Hexadecane, 2,6,11,15 tetramethyl; n-Hexadeconic acid; 9 Octadecenamide, (Z), have been reported as defrosting agent, antioxidant, flavour agent, antimicrobial, natural food additive, anti-inflammatory, anti-sleep disorder agent, respectively (Bharat et al., 2013).

All five bacterial strains secreted different metabolites. The metabolites, their retention time, peak area, area \% and peak height are represented in Table 3 and the GC-MS chromatograms are shown in Figure 1.

Total scans for L. plantarum DB-2 was 5709. Total run time was $19.42 \mathrm{~min}$ (5.00 - 24.42 min) (Figure 1a). The maximum peak area of
19659374.61 at the peak height of 7235909.56 was observed with $85.29 \%$ area covered at retention time $10.53 \mathrm{~min}$ (Table 3a, Figure $1 \mathrm{~b}$ ).

L. fermentum J-1 has total of 5713 scans. Total run time was $19.43 \mathrm{~min}$ (5.00 - 24.43 min) (Figure 1c). The maximum peak area of 863376582.00 at the peak height of 157335492.57 was observed with $54.03 \%$ area covered at retention time 11.37 min (Table 3b, Figure $1 d$ ).

P. acidilactici M-3 has total of 5715 scans. Total run time was $19.44 \mathrm{~min}$ (5.00 - 24.44 min) (Figure 1e). The maximum peak area of 31245106.39 at the peak height of 9971964.56 was observed with $58.41 \%$ area covered at retention time $10.70 \mathrm{~min}$ (Table 3c, Figure 1f).

L. plantarum SK-3 has total of 5719 scans. Total run time was $19.45 \mathrm{~min}$ (5.00 - 24.45 min) (Figure $1 \mathrm{~g}$ ). The maximum peak area of 55699133.56 at the peak height of 13468063.78 was observed with $92.21 \%$ area covered at retention time 10.49 min (Table 3d, Figure $1 \mathrm{~h}$ ).

P. pentosaceus SM-2 has total of 5860 scans. Total run time was $19.93 \mathrm{~min}(4.50-24.43$ min) (Figure 1i). The maximum peak area of 1586768.57 at the peak height of 739977.40 was observed with $68.57 \%$ area covered at retention time $10.13 \mathrm{~min}$ (Table 3e, Figure 1j).

Five metabolic compounds were distributed in four of the selected isolates viz. Isopropyl myristate; Methane, nitroso-; Propylene glycol; R-(-)-1,2 propanediol and Tetracosane. Compounds- Methane, nitroso-; Propylene glycol; R-(-)-1, 2 propanediol were present in L. plantarum DB-2, P. acidilactici M-3, L. plantarum SK-3 and P. pentosaceus SM-2. A metabolite Isopropyl myristate was secreted by $L$. fermentum $\mathrm{J}-1$, P. acidilactici M-3, L. plantarum SK-3 and P. pentosaceus SM-2. Compound - Tetracosane was reported in $L$. plantarum DB-2, L. fermentum $\mathrm{J}-1, P$. acidilactici $\mathrm{M}-3$ and $P$. pentosaceus SM2. Out of five metabolic compounds, Isopropyl myristate has been reported with medicinal activity against skin disorders. Out of 40, 3 metabolic compounds i.e. Eicosanoic acid, 2-Hydrazino ethanol and Tetradecanoic acid have been reported in three of the selected isolates. A metabolite - Eicosanoic acid was secreted by $L$. fermentum J-1, P. acidilactici $\mathrm{M}-3$, and P. pentosaceus SM-2. Compound - 2-Hydrazino ethanol was found in supernatant of 
Table 3. Metabolic profile of biocomponents identified in solvent extract of probiotic Lactic acid bacteria isolates by GC-MS

a) Lactobacillus plantarum DB-2

\begin{tabular}{lccc}
\hline $\begin{array}{l}\text { Retention } \\
\text { Time }\end{array}$ & $\begin{array}{c}\text { Peak } \\
\text { Area }\end{array}$ & $\begin{array}{c}\text { Area } \\
\%\end{array}$ & $\begin{array}{c}\text { Peak } \\
\text { Height }\end{array}$ \\
\hline 9.63 & 13668.39 & 0.06 & 23789.56 \\
9.65 & 5965.64 & 0.03 & 13938.29 \\
9.71 & 16405.98 & 0.07 & 28881.92 \\
10.09 & 13022.90 & 0.06 & 27200.06 \\
10.12 & 10917.74 & 0.05 & 18554.92 \\
10.36 & 532337.99 & 2.31 & 147518.20 \\
10.43 & 89582.31 & 0.39 & 148261.31 \\
10.53 & 19659374.61 & 85.29 & 7235909.56 \\
10.63 & 1922104.73 & 8.34 & 757552.20 \\
10.77 & 785784.33 & 3.41 & 319137.44 \\
\hline
\end{tabular}

(1)

Retention Metabolites

Time

\begin{tabular}{|c|c|c|c|}
\hline \multirow[t]{4}{*}{9.63} & Methane, nitroso- & $\mathrm{CH}_{3} \mathrm{NO}$ & $18501-20-7$ \\
\hline & Formamide & $\mathrm{CH}_{3}^{3} \mathrm{NO}$ & $75-12-7$ \\
\hline & Dodecane & $\mathrm{CH}_{3}\left(\mathrm{CH}_{2}\right)_{10} \mathrm{CH}_{3}$ & $112-40-3$ \\
\hline & DL-2,3-Butanediol & $\mathrm{C}_{4} \mathrm{H}_{10} \mathrm{O}_{2}$ & $6982-25-8$ \\
\hline \multirow[t]{3}{*}{9.65} & 2-Propanol, 1-hydrazino- & $\mathrm{C}_{3} \mathrm{H}_{10} \mathrm{~N}_{2} \mathrm{O}$ & $18501-20-7$ \\
\hline & Isopropyl alcohol & $\mathrm{C}_{3}^{3} \mathrm{H}_{8} \mathrm{O}^{2}$ & $67-63-0$ \\
\hline & Propylene glycol & $\mathrm{C}_{3} \mathrm{H}_{8} \mathrm{O}_{2}$ & $57-55-6$ \\
\hline \multirow[t]{3}{*}{9.71} & L-Lactic acid & $\mathrm{C}_{3} \mathrm{H}_{6} \mathrm{O}_{3}$ & $79-33-4$ \\
\hline & Acetic acid, anhydride with formic acid & $\mathrm{C}_{3} \mathrm{H}_{4} \mathrm{O}_{3}$ & $2258-42-6$ \\
\hline & R-(-)-1,2-propanediol & $\mathrm{C}_{3} \mathrm{H}_{8} \mathrm{O}_{2}$ & $4254-14-2$ \\
\hline \multirow[t]{3}{*}{10.09} & Methyltartronic acid & $\mathrm{C}_{4} \mathrm{H}_{6} \mathrm{O}_{5}^{2}$ & $595-98-2$ \\
\hline & Dodecanoic acid & $\mathrm{C}_{3} \mathrm{H}_{10} \mathrm{~N}_{2} \mathrm{O}$ & $18501-20-7$ \\
\hline & 2-Hydrazinoethanol & $\mathrm{C}_{2} \mathrm{H}_{8} \mathrm{~N}_{20}$ & $109-84-2$ \\
\hline \multirow[t]{3}{*}{10.12} & Tetradecane & $\mathrm{CH}_{2}\left(\mathrm{CH}_{3}\right)_{10} \mathrm{CH}_{2}$ & $629-59-4$ \\
\hline & Benzoic acid & $\mathrm{C}_{6} \mathrm{H}_{5} \mathrm{COOH}$ & $65-85-0$ \\
\hline & Undecane & $\mathrm{C}_{11}^{0} \mathrm{H}_{24}$ & $1120-21-4$ \\
\hline \multirow[t]{3}{*}{10.36} & Hexadecane, $2,6,11,15$, tetrame-thyl & $\mathrm{C}_{20} \mathrm{H}_{42}^{24}$ & $504-44-9$ \\
\hline & Benzaldehyde, 2,4, dimethyl & $\mathrm{C}_{9} \mathrm{H}_{10} \mathrm{O}$ & $15764-16-6$ \\
\hline & Phenol, 2,4 , bis (1,1 di-methylethyl) & $\mathrm{C}_{14} \mathrm{H}_{22} \mathrm{O}$ & $96-76-4$ \\
\hline \multirow[t]{3}{*}{10.43} & Decane & $\mathrm{C}^{10} \mathrm{H}_{22}^{22}$ & $124-18-5$ \\
\hline & Formamide & $\mathrm{CH}_{3} \mathrm{NO}$ & $75-12-7$ \\
\hline & Tetracosane & $\mathrm{C}_{24} \mathrm{H}_{5} \mathrm{O}$ & $646-31-1$ \\
\hline \multirow[t]{3}{*}{10.53} & Hexadecane & $\mathrm{C}_{16}^{24} \mathrm{H}_{34}$ & $544-76-3$ \\
\hline & Geranyl isovalerate & $\mathrm{C}_{15} \mathrm{H}_{26} \mathrm{O}_{2}$ & $109-20-6$ \\
\hline & $\begin{array}{l}\text { Pyrrolo [1,2a] pyrazine } 1,4 \text { dio-ne, } \\
\text { hexahydro } 3 \text { ( } 2 \text { methylpro-pyl) }\end{array}$ & $\mathrm{C}_{11} \mathrm{H}_{18} \mathrm{~N}_{2} \mathrm{O}_{2}$ & $5654-86-4$ \\
\hline \multirow[t]{3}{*}{10.63} & n-Hexadeconic acid & $\mathrm{C}_{16} \mathrm{H}_{32} \mathrm{O}_{2}$ & $57-10-3$ \\
\hline & 9 Octadecenamide, (Z) & $\mathrm{C}_{18} \mathrm{H}_{35} \mathrm{NO}$ & $301-02-0$ \\
\hline & L-Lactic acid & $\mathrm{C}_{3} \mathrm{H}_{6} \mathrm{O}_{3}$ & $79-33-4$ \\
\hline \multirow[t]{3}{*}{10.77} & Methane, nitroso- & $\mathrm{CH}_{3}^{3} \mathrm{NO}^{3}$ & $865-40-7$ \\
\hline & Ethylamine & $\mathrm{C}_{2} \mathrm{H}_{7} \mathrm{~N}$ & $75-04-7$ \\
\hline & 2-Ethoxyethylamine & $\mathrm{C}_{4}^{2} \mathrm{H}_{11} \mathrm{NO}$ & $110-76-9$ \\
\hline
\end{tabular}

L. plantarum DB-2, P. acidilactici $\mathrm{M}-3$ and P. pentosaceus SM-2. A metabolic compound Tetradecanoic acid was present in L. fermentum $\mathrm{J}-1, P$. acidilactici $\mathrm{M}-3$ and $P$. pentosaceus SM-2 (Table 2).

Six out of 40 metabolic compounds viz. DL-2, 3-Butanediol; Ethanamine, 2-propoxy-; Ethanol, 2-(2-propenyloxy); Hydroperoxide, pentyl; Methyltartronic acid and Squalene were present in two of the selected isolates. Metabolites - DL-2, 3-Butanediol and Methyltartronic acid were secreted by $L$. plantarum DB-2 and L. plantarum SK-3. Compounds - Ethanamine, 2-propoxy-; Ethanol, 2-(2-propenyloxy) and Hydroperoxide, pentyl were found in the supernatant of P. acidilactici M-3 and L. plantarum SK-3.
Molecular formula Cas No. 
Metabolite - Squalene was synthesized in the culture supernatant of $P$. acidilactici $\mathrm{M}-3$ and P. pentosaceus SM-2 (Table 2). Squalene is a triterpine compound, originally found in shark liver oil and has a high therapeutic potential. Squalene is a therapeutic agent having anti-cancerous, anti-tumour, chemo-preventive, antioxidant, and sunscreen properties along with anti-microbial activity (Ezhilan and Neelamegam, 2012). In industry, it is scarcely produced by plant sources along with the shark liver oil. Microbial production

b) Lactobacillus fermentum J-1

\begin{tabular}{lccc}
\hline $\begin{array}{l}\text { Retention } \\
\text { Time }\end{array}$ & $\begin{array}{c}\text { Peak } \\
\text { Area }\end{array}$ & $\begin{array}{c}\text { Area } \\
\%\end{array}$ & $\begin{array}{c}\text { Peak } \\
\text { Height }\end{array}$ \\
\hline 5.04 & 2763275.83 & 0.17 & 4173857.72 \\
11.30 & 152563796.70 & 9.55 & 141697072 \\
11.37 & 863376582.00 & 54.03 & 157335492.57 \\
11.53 & 497177369.61 & 31.12 & 129599071.95 \\
11.61 & 81982240.17 & 5.13 & 26851053.15 \\
\hline
\end{tabular}

Retention Metabolites
Time

of Squalene is an attractive alternative to label the issue with an objective to increase its productivity and purity by using biotechnological interventions. Although, a detailed study is required for the commercialization of the production of Squalene from native probiotic bacteria.

9 metabolites out of 40 were produced by only one selected isolate viz. Acetic acid, anhydride with formic acid; 4-amino-1-butanol; 2-Ethoxyethylamine; Ethylamine; Formamide; Hydroperoxide, heptyl; Hydroperoxide, 1-methylhexyl; 4-Penten-2-ol and Propanoic acid, 2-hydroxy-, methylester. Compounds - Acetic acid, anhydride with formic acid; 2-Ethoxyethylamine; Ethylamine; Formamide were produced by $L$. plantarum DB-2. Metabolite - 4-amino-1-butanol was found to be synthesized by $L$. plantarum SK-3. Metabolite - Hydroperoxide, heptyl was present in P. acidilactici M-3. Compounds Hydroperoxide, 1-methylhexyl and Propanoic acid, 2-hydroxy-, methylester were secreted

Molecular Cas No.

Formula

\begin{tabular}{|c|c|c|c|}
\hline \multirow[t]{5}{*}{5.04} & Hydroperoxide, pentyl & $\mathrm{C}_{5} \mathrm{H}_{12} \mathrm{O}_{2}$ & $74-80-6$ \\
\hline & Ethanol, 2-(2-propenyloxy)- & $\mathrm{C}_{5} \mathrm{H}_{10} \mathrm{O}_{2}^{2}$ & $111-45-5$ \\
\hline & Hydroperoxide, 1-methylhexyl & $\mathrm{C}_{7} \mathrm{H}_{16} \mathrm{O}_{2}$ & $762-46-9$ \\
\hline & Decane & $\mathrm{C}_{10} \mathrm{H}_{22}$ & $124-18-5$ \\
\hline & Undecane & $\mathrm{C}_{11} \mathrm{H}_{24}$ & $1120-21-4$ \\
\hline \multirow[t]{5}{*}{11.30} & Benzaldehyde, 2,4, dimethyl & $\mathrm{C}_{9} \mathrm{H}_{10} \mathrm{O}$ & $15764-16-6$ \\
\hline & Benzoic acid & $\mathrm{C}_{6} \mathrm{H}_{5} \mathrm{COOH}$ & $65-85-0$ \\
\hline & 2-Propanol, 1-hydrazino- & $\mathrm{C}_{3}^{0} \mathrm{H}_{10}^{5} \mathrm{~N}_{2} \mathrm{O}$ & $18501-20-7$ \\
\hline & Isopropyl alcohol & $\mathrm{C}_{3} \mathrm{H}_{8} \mathrm{O}$ & $67-63-0$ \\
\hline & L-Lactic acid & $\mathrm{C}_{3} \mathrm{H}_{6} \mathrm{O}_{3}$ & $79-33-4$ \\
\hline \multirow[t]{5}{*}{11.37} & Dodecane & $\mathrm{C}_{12} \mathrm{H}_{26}$ & $112-40-3$ \\
\hline & Phenol, 2,4 bis (1,1dimethylethyl) & $\mathrm{C}_{14} \mathrm{H}_{22} \mathrm{O}$ & $96-76-4$ \\
\hline & Propanoic acid, 2-hydroxy-, methyl ester, (ñ)- & $\mathrm{C}_{4} \mathrm{H}_{8} \mathrm{O}_{3}$ & $2155-30-8$ \\
\hline & Dodecanoic acid & $\mathrm{C}_{12} \mathrm{H}_{24} \mathrm{O}_{2}$ & $143-07-7$ \\
\hline & Tetradecane & $\mathrm{CH}_{2}\left(\mathrm{CH}_{3}\right)_{10} \mathrm{CH}_{2}$ & $629-59-4$ \\
\hline \multirow[t]{5}{*}{11.53} & Hexadecane & $\mathrm{C}_{16} \mathrm{H}_{34}$ & $544-76-3$ \\
\hline & Geranyl Isovalerate & $\mathrm{C}_{15} \mathrm{H}_{26} \mathrm{O}_{2}$ & $109-20-6$ \\
\hline & $\begin{array}{l}\text { Pyrrolo [ } 1,2 \text { a ] pyrazine } 1,4 \text { dione, } \\
\text { hexahydro } 3 \text { ( } 2 \text { methylpropyl) }\end{array}$ & $\mathrm{C}_{11} \mathrm{H}_{18} \mathrm{~N}_{2} \mathrm{O}_{2}$ & $5654-86-4$ \\
\hline & n-Hexadeconic acid & $\mathrm{C}_{16} \mathrm{H}_{32} \mathrm{O}_{2}$ & $57-10-3$ \\
\hline & 2-Hydrazinoethanol & $\mathrm{C}_{2} \mathrm{H}_{8} \mathrm{~N}_{2} \mathrm{O}$ & $109-84-2$ \\
\hline \multirow[t]{6}{*}{11.61} & Eicosanoic acid & $\mathrm{C}_{20} \mathrm{H}_{40} \mathrm{O}_{2}$ & $506-30-9$ \\
\hline & Tetracosane & $\mathrm{C}_{24} \mathrm{H}_{50}$ & $646-31-1$ \\
\hline & Isopropyl myristate & $\mathrm{C}_{17}^{24} \mathrm{H}_{34} \mathrm{O}_{2}$ & $110-27-0$ \\
\hline & 9 Octadecenamide, (Z) & $\mathrm{C}_{18} \mathrm{H}_{35} \mathrm{NO}^{2}$ & $301-02-0$ \\
\hline & Tetradecanoic acid & $\mathrm{C}_{14} \mathrm{H}_{28} \mathrm{O}_{2}$ & $544-63-8$ \\
\hline & Hexadecane, 2,6,11,15 tetrame-thyl & $\mathrm{C}_{20} \mathrm{H}_{42}$ & $504-44-9$ \\
\hline
\end{tabular}


by L. fermentum J1. Metabolite - 4-Penten2-ol was produced by $P$. pentosaceus SM-2 (Table 2).

Probiotics has a vital role in well-being and health beyond basic nutrition. Probiotics have potential health benefits such as antimicrobial activity, antimutagenic, anticarcinogenic activities,

c) Pediococcus acidilactici M-3

\begin{tabular}{lccc}
\hline $\begin{array}{l}\text { Retention } \\
\text { Time }\end{array}$ & $\begin{array}{c}\text { Peak } \\
\text { Area }\end{array}$ & $\begin{array}{c}\text { Area } \\
\%\end{array}$ & $\begin{array}{c}\text { Peak } \\
\text { Height }\end{array}$ \\
\hline 5.02 & 3774941.17 & 7.06 & 3718077.48 \\
10.58 & 2981495.15 & 5.57 & 1994601.20 \\
10.63 & 1773985.26 & 3.32 & 1572423.15 \\
10.70 & 31245106.39 & 58.41 & 9971964.56 \\
10.80 & 9650577.59 & 18.04 & 3715383.92 \\
11.00 & 4064008.32 & 7.60 & 1071788.04 \\
\hline
\end{tabular}

Retention Metabolites
Time

\begin{tabular}{|c|c|c|c|}
\hline \multirow[t]{4}{*}{5.02} & Hydroperoxide, pentyl & $\mathrm{C}_{5} \mathrm{H}_{12} \mathrm{O}_{2}$ & $74-80-6$ \\
\hline & Hydroperoxide, heptyl & $\mathrm{C}_{7}^{5} \mathrm{H}_{16}^{12} \mathrm{O}_{2}^{2}$ & $764-81-8$ \\
\hline & Ethanol, 2-(2-propenyloxy)- & $\mathrm{C}_{5} \mathrm{H}_{10} \mathrm{O}_{2}^{2}$ & $111-45-5$ \\
\hline & Benzoic acid & $\mathrm{C}_{6} \mathrm{H}_{5} \mathrm{COOH}$ & $65-85-0$ \\
\hline \multirow[t]{5}{*}{10.58} & Dodecane & $\mathrm{C}_{12} \mathrm{H}_{26}$ & $112-40-3$ \\
\hline & Undecane & $\mathrm{C}_{11}^{12} \mathrm{H}_{24}$ & $1120-21-4$ \\
\hline & Isopropyl alcohol & $\mathrm{C}_{3} \mathrm{H}_{8} \mathrm{O}$ & $67-63-0$ \\
\hline & Propylene Glycol & $\mathrm{C}_{3} \mathrm{H}_{8} \mathrm{O}_{2}$ & $57-55-6$ \\
\hline & L-Lactic acid & $\mathrm{C}_{3}^{3} \mathrm{H}_{6}^{8} \mathrm{O}_{3}^{2}$ & $79-33-4$ \\
\hline \multirow[t]{5}{*}{10.63} & R-(-)-1,2-propanediol & $\mathrm{C}_{3} \mathrm{H}_{8} \mathrm{O}_{2}$ & $4254-14-2$ \\
\hline & Tetradecane & $\mathrm{CH}_{2}\left(\mathrm{CH}_{3}\right)_{10} \mathrm{CH}_{2}$ & $629-59-4$ \\
\hline & Phenol, 2,4 bis (1,1dimethylethyl) & $\mathrm{C}_{14} \mathrm{H}_{22} \mathrm{O}$ & $96-76-4$ \\
\hline & Hexadecane, 2,6,11,15 tetra-methyl & $\mathrm{C}_{20} \mathrm{H}_{42}$ & $504-44-9$ \\
\hline & Benzaldehyde, 2,4, dimethyl & $\mathrm{C}_{9} \mathrm{H}_{10} \mathrm{O}$ & $15764-16-6$ \\
\hline \multirow[t]{5}{*}{10.70} & 2-Propanol, 1-hydrazino- & $\mathrm{C}_{3} \mathrm{H}_{10} \mathrm{~N}_{2} \mathrm{O}$ & $18501-20-7$ \\
\hline & Dodecanoic acid & $\mathrm{C}_{12} \mathrm{H}_{24} \mathrm{O}_{2}$ & $143-07-7$ \\
\hline & Methane, nitroso- & $\mathrm{CH}_{3} \mathrm{NO}^{2}$ & $865-40-7$ \\
\hline & Tetracosane & $\mathrm{C}_{24} \mathrm{H}_{5} \mathrm{O}$ & $646-31-1$ \\
\hline & Decane & $\mathrm{C}_{10}^{24} \mathrm{H}_{22}$ & $124-18-5$ \\
\hline \multirow[t]{5}{*}{10.80} & Eicosanoic acid & $\mathrm{C}_{20} \mathrm{H}_{40} \mathrm{O}_{2}$ & $506-30-9$ \\
\hline & Hexadecane & $\mathrm{C}_{16} \mathrm{H}_{34}$ & $544-76-3$ \\
\hline & Isopropyl myristate & $\mathrm{C}_{17} \mathrm{H}_{34} \mathrm{O}_{2}$ & $110-27-0$ \\
\hline & Geranyl isovalerate & $\mathrm{C}_{15} \mathrm{H}_{26} \mathrm{O}_{2}$ & $109-20-6$ \\
\hline & n-Hexadeconic acid & $\mathrm{C}_{16} \mathrm{H}_{32} \mathrm{O}_{2}^{2}$ & $57-10-3$ \\
\hline \multirow[t]{5}{*}{11.00} & Ethanamine, 2-propoxy- & & \\
\hline & $\begin{array}{l}\text { Pyrrolo [ } 1,2 \text { a ] pyrazine } 1,4 \text { dione, } \\
\text { hexahydro } 3 \text { ( } 2 \text { methylpropyl) }\end{array}$ & $\mathrm{C}_{11} \mathrm{H}_{18} \mathrm{~N}_{2} \mathrm{O}_{2}$ & $5654-86-4$ \\
\hline & Tetradecanoic acid & $\mathrm{C}_{14} \mathrm{H}_{28} \mathrm{O}_{2}$ & $544-63-8$ \\
\hline & 9 Octadecenamide, (Z) & $\mathrm{C}_{18}^{14} \mathrm{H}_{35}^{28} \mathrm{NO}$ & $301-02-0$ \\
\hline & Squalene & $\mathrm{C}_{30} \mathrm{H}_{5} \mathrm{O}$ & $111-02-4$ \\
\hline
\end{tabular}

constipation, regulation of immune function, improving gastrointestinal health, reducing lactose intolerance, and allergenic diseases such as food allergy, etc. Due to these health benefits, probiotic bacteria and their metabolites are gaining importance in pharmaceutical preparation, food products, medicines and dietary supplements (Da Cruz et al., 2009; Settanni and Moschetti, 2010; Fernandez et al., 2013). Production of different primary and secondary metabolites by the probiotic bacteria, employs their biological effects directly or by modifying the immune system. The metabolites of lactic acid bacteria provide therapeutic benefits in preventing and curing various diseases and also responsible for the development of flavour, aroma and texture in food products (Chen et al., 2014). The metabolites produced by lactic acid bacteria in this study

Molecular Cas No.

Formula

$\mathrm{C}_{7} \mathrm{H}_{16} \mathrm{O}_{2}$

67-63-0

$57-55-6$

96-76-4

$504-44-9$

46-31-1

124-18-5

109-20-6

57-10-3

$\mathrm{C}_{11} \mathrm{H}_{18} \mathrm{~N}_{2} \mathrm{O}_{2} \quad 5654-86-4$

$\mathrm{C}_{14} \mathrm{H}_{28} \mathrm{O}_{2} \quad$ 544-63-8

301-02-0

www.microbiologyjournal.org 
have been remarked with various functional as well as therapeutic properties viz. food additives, flavouring agents, anti-inflammatory, anti-cancerous, potential against skin disorders, cholesterol-lowering and anti-diarrheal properties and endorse their huge potential in food and pharmaceutical industries.

Many researchers have observed similar metabolic products in many lactic acid bacteria influencing human metabolism when incorporated into the system as probiotics or the food fermented by the respective bacteria. Cagno et al. (2009) observed tomato juice fermented

d) Lactobacillus plantarum SK-3

\begin{tabular}{lccc}
\hline $\begin{array}{l}\text { Retention } \\
\text { Time }\end{array}$ & $\begin{array}{c}\text { Peak } \\
\text { Area }\end{array}$ & $\begin{array}{c}\text { Area } \\
\%\end{array}$ & $\begin{array}{c}\text { Peak } \\
\text { Height }\end{array}$ \\
\hline 9.75 & 625128.64 & 1.03 & 279968.97 \\
10.33 & 87526.28 & 0.14 & 137242.54 \\
10.42 & 1067849.36 & 1.77 & 1455637.02 \\
10.45 & 898971.70 & 1.49 & 1508377.52 \\
10.49 & 55699133.56 & 92.21 & 13468063.78 \\
10.69 & 2027689.17 & 3.36 & 774610.72 \\
\hline
\end{tabular}

Retention $\quad$ Metabolites
Time

\begin{tabular}{ll}
\hline 9.75 & Isopropyl alcohol \\
& L-Lactic acid \\
& 2-propanol, 1-hydrazino \\
& Benzoic acid \\
& 2-Hydrazinoethanol \\
& Dodecane \\
& Benzaldehyde, 2,4, dimethyl \\
& Undecane \\
& Propylene glycol \\
& Tetradecane \\
& Dodecanoic acid \\
& Hexadecane, 2,6,11,15 tetra-methyl \\
& R-(-)-1,2-propanediol \\
& Hexadecane \\
& Isopropyl myristate \\
& Decane \\
& Methane, nitroso- \\
& Phenol, 2,4 bis (1,1dimethylethyl) \\
& Geranyl isovalerate \\
& n-Hexadeconic acid \\
& Ethanamine, 2-propoxy- \\
& 4-Amino-1-butanol \\
& Pyrrolo [ 1,2 a ] pyrazine 1,4 dione, \\
& hexahydro 3 (2 methylpropyl) \\
& 9 Octadecenamide, (Z) \\
& \\
& \\
& \\
&
\end{tabular}

Molecular

Formula

with L. plantarum POM1 and POM35 for the effect of metabolites produced by the bacterial starters (by GC-MS technique) on the sensory and health-promoting properties of tomato juice and found a large number of volatile compounds in fermented tomato juice as compared to the control. Lee et al. (2009) isolated the metabolites from lactic acid bacteria in grape wines through GC-based metabolic profiling and ${ }^{1} \mathrm{H}-\mathrm{NMR}$ and identified various organic acids such as lactic acid, tartaric acid and volatile compounds such as benzoic acid, dodecanoic acid, dodecamethyl cyclohexasiloxane, isopropyl myristate, tetradecanoic acid, 1-hexadecanol, hexadeconoic acid and octadecanoic acid responsible for wine odour and flavour. Vanaja et al. (2011) observed the metabolites secreted by L. plantarum LPcfr and found compounds viz. hexadecane, hexadeconic acid, dodecanal having antifungal and antioxidant properties and fatty acids such as stearic and palmitic acids having antifungal properties. Sheela et al. (2012) found antimicrobial compounds (fatty acids such as palmitic acid and stearic acid and

$\begin{array}{ll}\mathrm{C}_{3} \mathrm{H}_{8} \mathrm{O} & 67-63-0 \\ \mathrm{C}_{3} \mathrm{H}_{6} \mathrm{O}_{3} & 79-33-4 \\ \mathrm{C}_{3} \mathrm{H}_{10} \mathrm{~N}_{2} \mathrm{O} & 18501-20-7 \\ \mathrm{C}_{6} \mathrm{H}_{5} \mathrm{COOH} & 65-85-0 \\ \mathrm{C}_{2} \mathrm{H}_{8} \mathrm{~N}_{2} \mathrm{O} & 109-84-2 \\ \mathrm{C}_{12} \mathrm{H}_{26} & 112-40-3 \\ \mathrm{C}_{9} \mathrm{H}_{10} \mathrm{O} & 15764-16-6 \\ \mathrm{C}_{11} \mathrm{H}_{24} & 1120-21-4 \\ \mathrm{C}_{3} \mathrm{H}_{8} \mathrm{O}_{2} & 57-55-6 \\ \mathrm{CH}_{2}\left(\mathrm{CH}_{3}\right)_{10} \mathrm{CH}_{2} & 629-59-4 \\ \mathrm{C}_{12} \mathrm{H}_{24} \mathrm{O}_{2} & 143-07-7 \\ \mathrm{C}_{22} \mathrm{H}_{42} & 504-44-9 \\ \mathrm{C}_{3} \mathrm{H}_{8} \mathrm{O}_{2} & 4254-14-2 \\ \mathrm{C}_{16} \mathrm{H}_{34} & 544-76-3 \\ \mathrm{C}_{17} \mathrm{H}_{34} \mathrm{O}_{2} & 110-27-0 \\ \mathrm{C}_{110} \mathrm{H}_{22} & 124-18-5 \\ \mathrm{CH}_{3} \mathrm{NO} & 865-40-7 \\ \mathrm{C}_{14} \mathrm{H}_{22} \mathrm{O} & 96-76-4 \\ \mathrm{C}_{15} \mathrm{H}_{26} \mathrm{O}_{2} & 109-20-6 \\ \mathrm{C}_{16} \mathrm{H}_{32} \mathrm{O}_{2} & 57-10-3 \\ \mathrm{C}_{5} \mathrm{H}_{13} \mathrm{NO} & 42185-03-5 \\ \mathrm{C}_{4} \mathrm{H}_{11} \mathrm{NO} & 13325-10-5 \\ \mathrm{C}_{11} \mathrm{H}_{18} \mathrm{~N}_{2} \mathrm{O}_{2} & 5654-86-4 \\ \mathrm{C}_{18} \mathrm{H}_{35} \mathrm{NO} & \\ \mathrm{C}_{35} & 301-02-0\end{array}$


Phenol, 2, 4-bis (1, dimethylethyl)) after GC-MS in symbiotic cow milk beverage fermented with L. kefiranofaciens, Candida kefir and Saccharomyces boulardii. The fermented cow milk symbiotic beverage had the greater anti-diarrhoeal effect as compared to other milk beverages, thereby indicating the role of antibacterial metabolites produced by the probiotic bacteria. Padmavathi

e) Pediococcus pentosaceus SM-2

\begin{tabular}{lccc}
\hline $\begin{array}{l}\text { Retention } \\
\text { Time }\end{array}$ & $\begin{array}{c}\text { Peak } \\
\text { Area }\end{array}$ & $\begin{array}{c}\text { Area } \\
\%\end{array}$ & $\begin{array}{c}\text { Peak } \\
\text { Height }\end{array}$ \\
\hline 9.72 & 216757.74 & 9.37 & 98425.02 \\
9.79 & 210048.41 & 9.08 & 89220.41 \\
9.82 & 72569.37 & 3.14 & 142459.44 \\
9.86 & 36228.30 & 1.57 & 51748.50 \\
10.13 & 1586768.57 & 68.57 & 739977.40 \\
10.20 & 191626.29 & 8.28 & 115859.62 \\
\hline
\end{tabular}

Retention Metabolites
Time

\begin{tabular}{|c|c|c|c|}
\hline \multirow[t]{4}{*}{9.72} & Methyltartronic acid & $\mathrm{C}_{4} \mathrm{H}_{6} \mathrm{O}_{5}$ & $595-98-2$ \\
\hline & L-Lactic acid & $\mathrm{C}_{3}^{4} \mathrm{H}_{6} \mathrm{O}_{3}^{5}$ & $79-33-4$ \\
\hline & 2-propanol, 1-hydrazino & $\mathrm{C}_{3}^{3} \mathrm{H}_{10}^{6} \mathrm{~N}_{2} \mathrm{O}$ & $18501-20-7$ \\
\hline & Benzoic acid & $\mathrm{C}_{6}^{3} \mathrm{H}_{5} \mathrm{COOH}$ & $65-85-0$ \\
\hline \multirow[t]{5}{*}{9.79} & R-(-)-1,2-propanediol & $\mathrm{C}_{3} \mathrm{H}_{8} \mathrm{O}_{2}$ & $4254-14-2$ \\
\hline & DL- 2,3 -Butanediol & $\mathrm{C}_{4}^{3} \mathrm{H}_{10}^{8} \mathrm{O}_{2}^{2}$ & $6982-25-8$ \\
\hline & Dodecane & $\mathrm{C}_{12} \mathrm{H}_{26}$ & $112-40-3$ \\
\hline & Decane & $\mathrm{C}_{10} \mathrm{H}_{22}$ & $124-18-5$ \\
\hline & Benzaldehyde, 2,4, dimethyl & $\mathrm{C}_{9} \mathrm{H}_{10} \mathrm{O}$ & $15764-16-6$ \\
\hline \multirow[t]{5}{*}{9.82} & Propylene glycol & $\mathrm{C}_{3}^{9} \mathrm{H}_{8} \mathrm{O}_{2}$ & $57-55-6$ \\
\hline & Tetradecane & $\mathrm{CH}_{2}^{3}\left(\mathrm{CH}_{3}\right)_{10} \mathrm{CH}_{2}$ & $629-59-4$ \\
\hline & Dodecanoic acid & $\mathrm{C}_{12} \mathrm{H}_{24} \mathrm{O}_{2}$ & $143-07-7$ \\
\hline & Phenol, 2,4 bis (1,1dimethylethyl) & $\mathrm{C}_{14} \mathrm{H}_{22} \mathrm{O}$ & $96-76-4$ \\
\hline & Hexadecane, 2,6,11,15 tetra-methyl & $\mathrm{C}_{20}^{14} \mathrm{H}_{42}$ & $504-44-9$ \\
\hline \multirow[t]{5}{*}{9.86} & Isopropyl Alcohol & $\mathrm{C}_{3} \mathrm{H}_{8} \mathrm{O}$ & $67-63-0$ \\
\hline & Hexadecane & $\mathrm{C}_{16}^{3} \mathrm{H}_{34}$ & $544-76-3$ \\
\hline & Tetracosane & $\mathrm{C}_{24}^{10} \mathrm{H}_{50}$ & $646-31-1$ \\
\hline & Tetradecanoic acid & $\mathrm{C}_{14}^{24} \mathrm{H}_{28}^{50} \mathrm{O}_{2}$ & $544-63-8$ \\
\hline & Undecane & $\mathrm{C}_{11}^{14} \mathrm{H}_{24}$ & $1120-21-4$ \\
\hline \multirow[t]{5}{*}{10.13} & Methane, nitroso- & $\mathrm{CH}_{3} \mathrm{NO}$ & $865-40-7$ \\
\hline & Isopropyl myristate & $\mathrm{C}_{17} \mathrm{H}_{34} \mathrm{O}_{2}$ & $110-27-0$ \\
\hline & Geranyl isovalerate & $\mathrm{C}_{15} \mathrm{H}_{26} \mathrm{O}_{2}^{2}$ & $109-20-6$ \\
\hline & n-Hexadeconic acid & $\mathrm{C}_{16} \mathrm{H}_{32} \mathrm{O}_{2}^{2}$ & $57-10-3$ \\
\hline & Eicosanoic acid & $\mathrm{C}_{20} \mathrm{H}_{4} \mathrm{OO}_{2}$ & $506-30-9$ \\
\hline \multirow[t]{4}{*}{10.20} & 4-Penten-2-ol & $\mathrm{C} 5 \mathrm{H} 10 \mathrm{O}^{2}$ & $625-31-0$ \\
\hline & $\begin{array}{l}\text { Pyrrolo [ } 1,2 \text { a ] pyrazine } 1,4 \text { dione, } \\
\text { hexahydro } 3 \text { ( } 2 \text { methylpropyl) }\end{array}$ & $\mathrm{C}_{11} \mathrm{H}_{18} \mathrm{~N}_{2} \mathrm{O}_{2}$ & $5654-86-4$ \\
\hline & 9 Octadecenamide, (Z) & $\mathrm{C}_{18} \mathrm{H}_{35} \mathrm{NO}$ & $301-02-0$ \\
\hline & Squalene & $\mathrm{C}_{30} \mathrm{H}_{5} \mathrm{O}$ & $111-02-4$ \\
\hline
\end{tabular}

et al. (2014) studied the anti-biofilm efficacy and anti-quorum sensing of the metabolites produced by marine Vibrio alginolyticus G16 against Serratia marcescens and found the active compound as phenol, 2, 4-bis (1,1-dimethylethyl) after purification and mass spectrometric analysis. Sheela et al. (2015) studied the biocontrol efficacy of symbiotic strawberry juice and found most of the metabolites were of antibacterial, anti-diarrhoeal (17-pentatriacontene) and of antioxidative nature. Effect of probiotics and prebiotics was evaluated on the metabolites isolated from human microbiota and found these metabolites helping in the regulation of colonic cell proliferation, inactivation of toxic compounds and enhancement of health-promoting properties (Vitali et al., 2012). Hong-Xin et al. (2015) used GC-MS analysis to identify aroma compounds viz. decane, dodecanoic acid, hexadecanoic acid, hexanoic acid 4-hexadecyl ester and phenol 2,4

Molecular Cas No.

Formula

www.microbiologyjournal.org 
bis (1,1-dimethylethyl) during ripening period (180 days) of cheese fermented with L. casei LC2W and found these metabolites help in accelerating the ripening process.
The reliability of the technique in investigating the effect of metabolic compounds on colonic metabolic signature has been recently

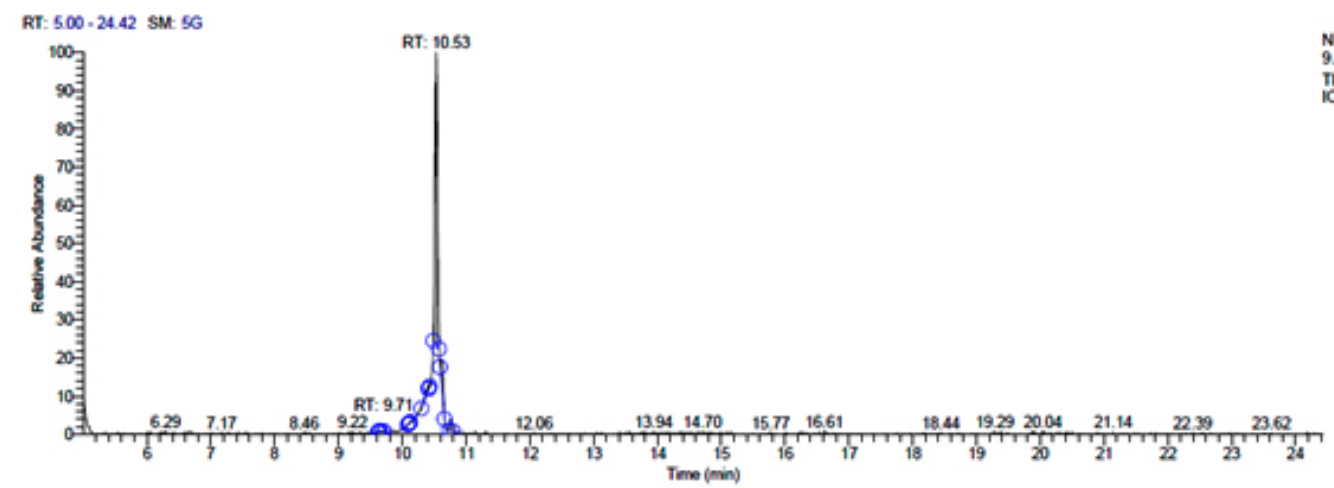

(a) GC-MS chromatogram of metabolites secreted by L. plantarum DB-2

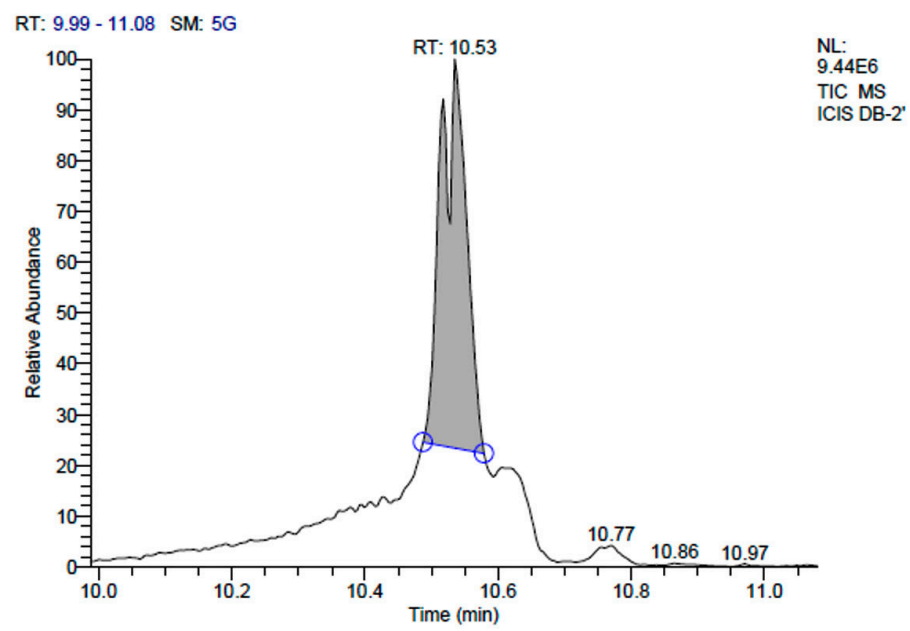

(b) GC-MS chromatogram of L. plantarum DB-2 at retention time $10.53 \mathrm{~min}$

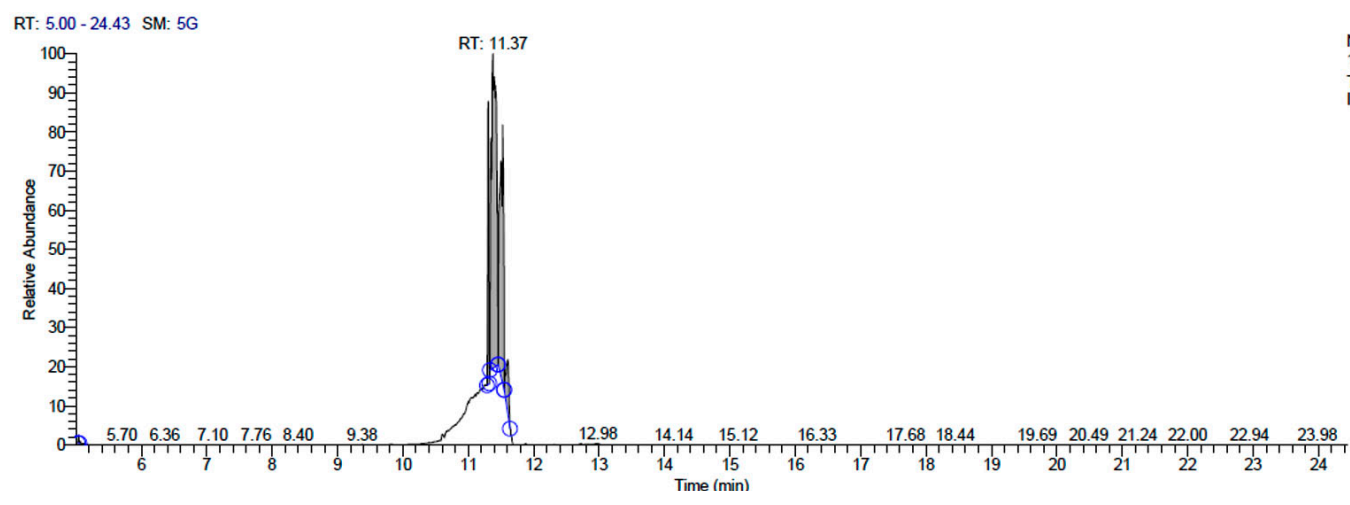

(c) GC-MS chromatogram of metabolites secreted by L. fermentum J-1 


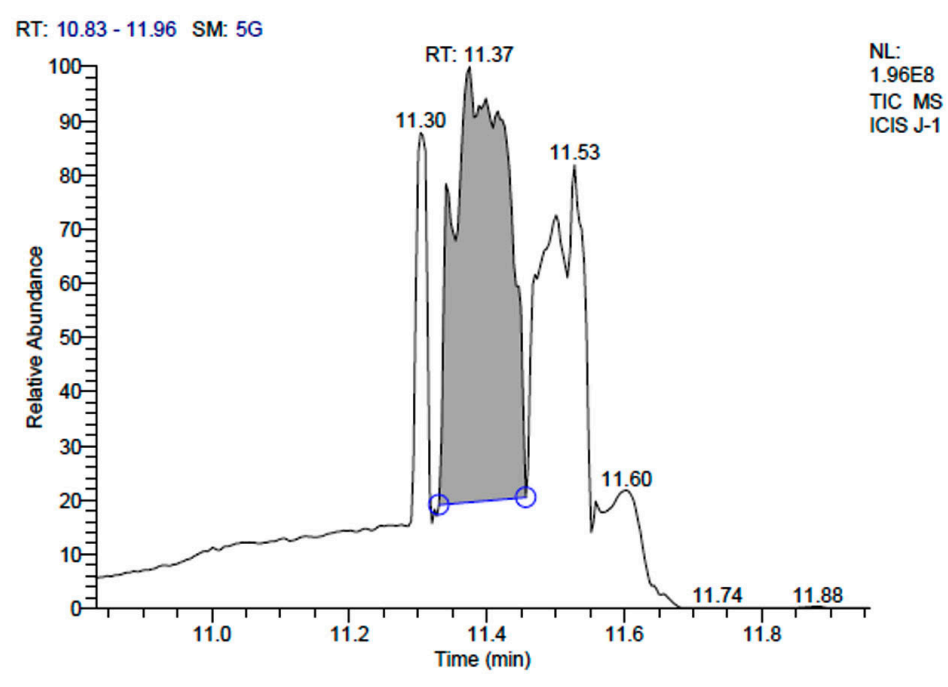

(d) GC-MS chromatogram of L. fermentum J-1 at retention time $11.37 \mathrm{~min}$

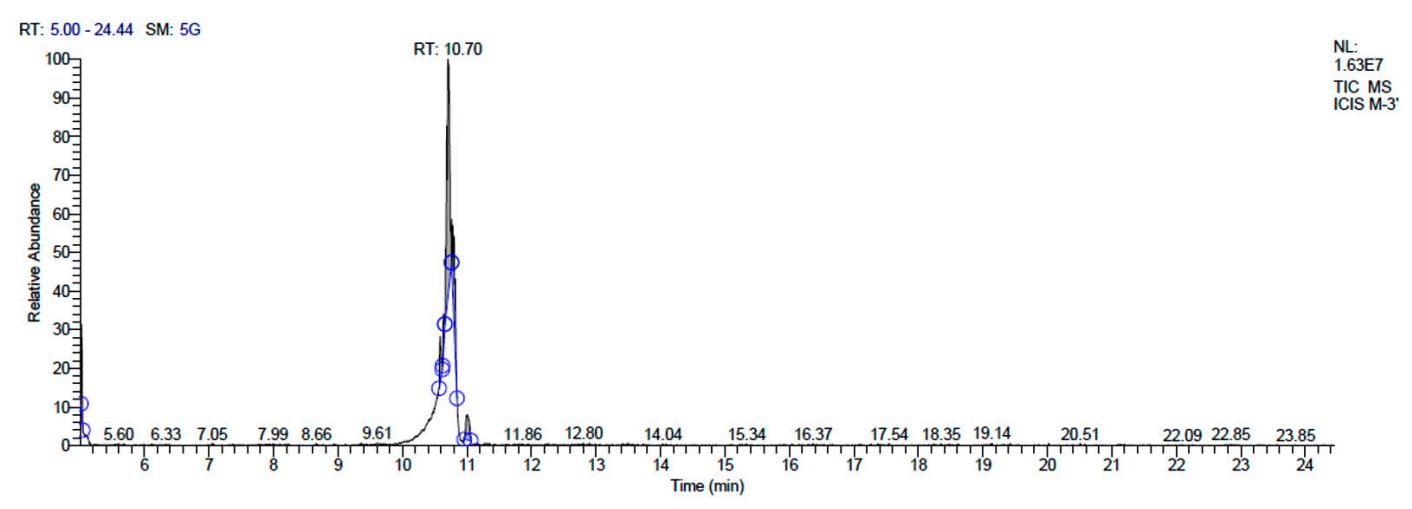

(e) GC-MS chromatogram of metabolites secreted by $P$. acidilactici M-3

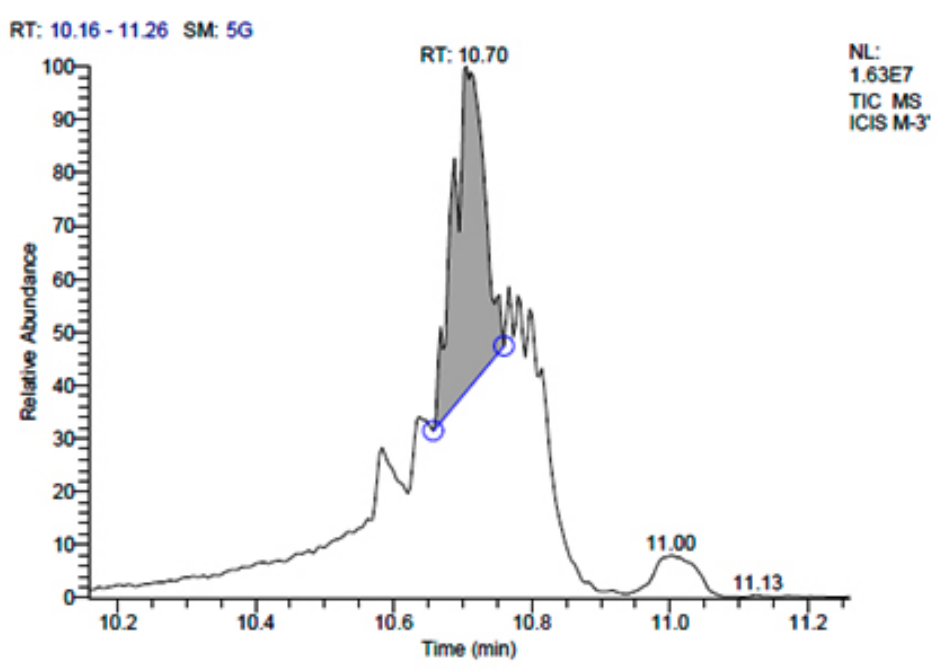

(f) GC-MS chromatogram of $P$. acidilactici M-3 at retention time $10.70 \mathrm{~min}$ 


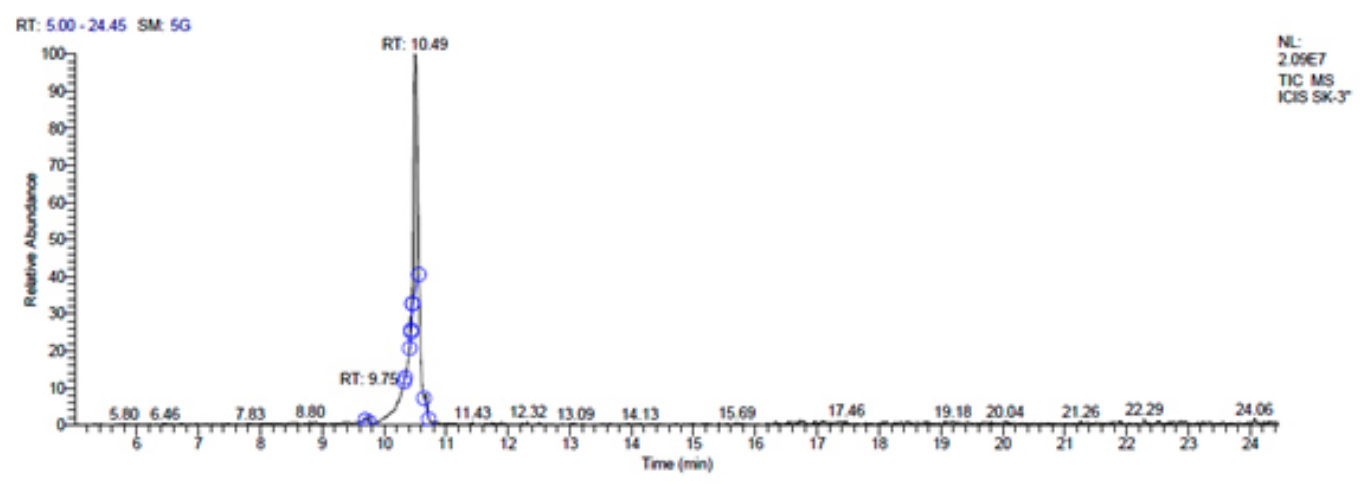

(g) GC-MS chromatogram of metabolites secreted by L. plantarum SK-3

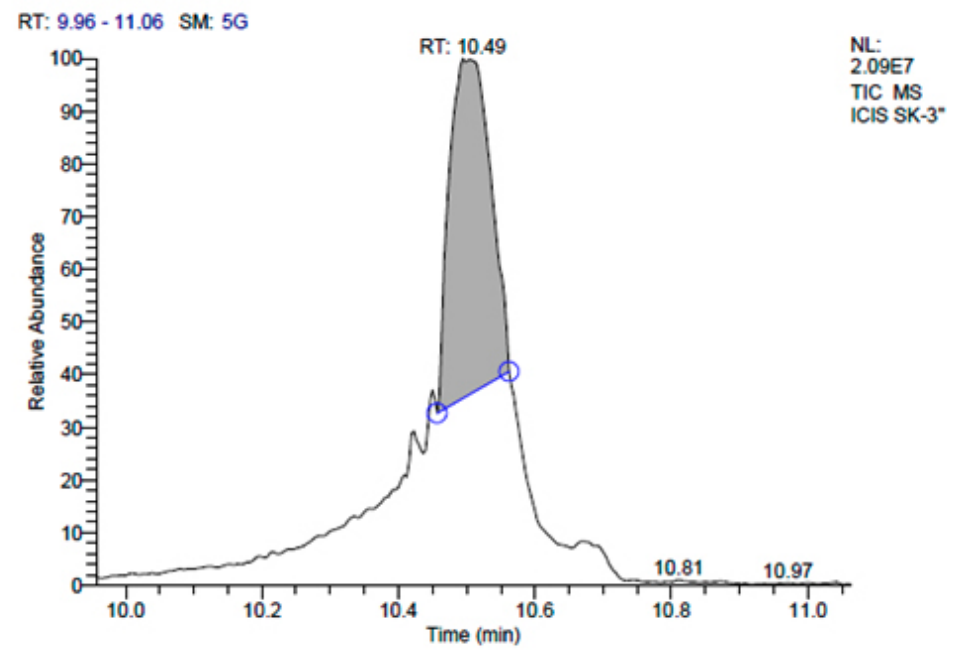

(h) GC-MS chromatogram of L. plantarum SK-3 at retention time $10.49 \mathrm{~min}$

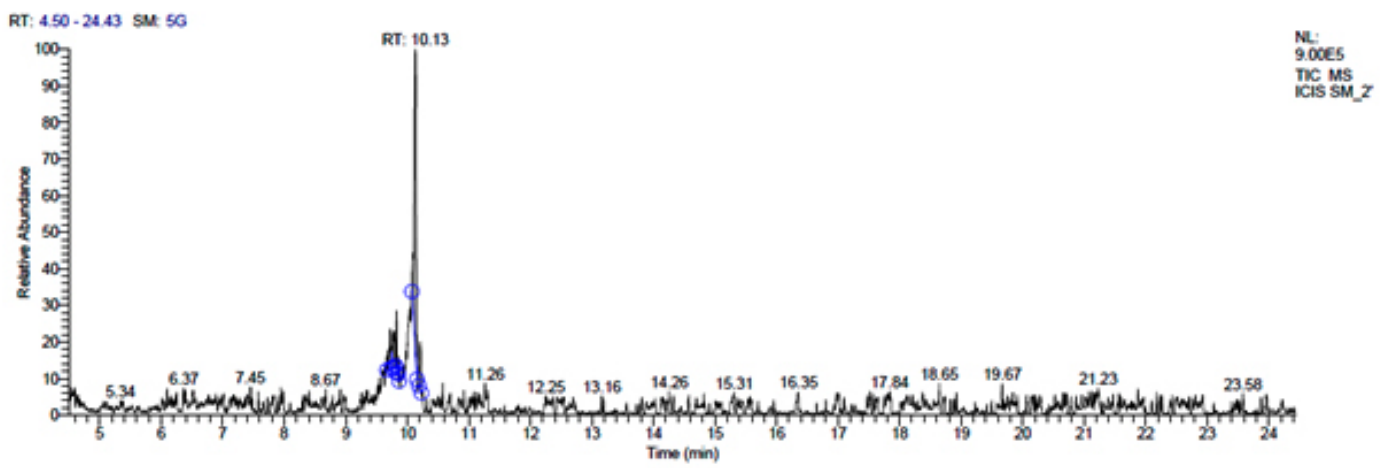

(i) GC-MS chromatogram of metabolites secreted by P. pentosaceus SM-2 


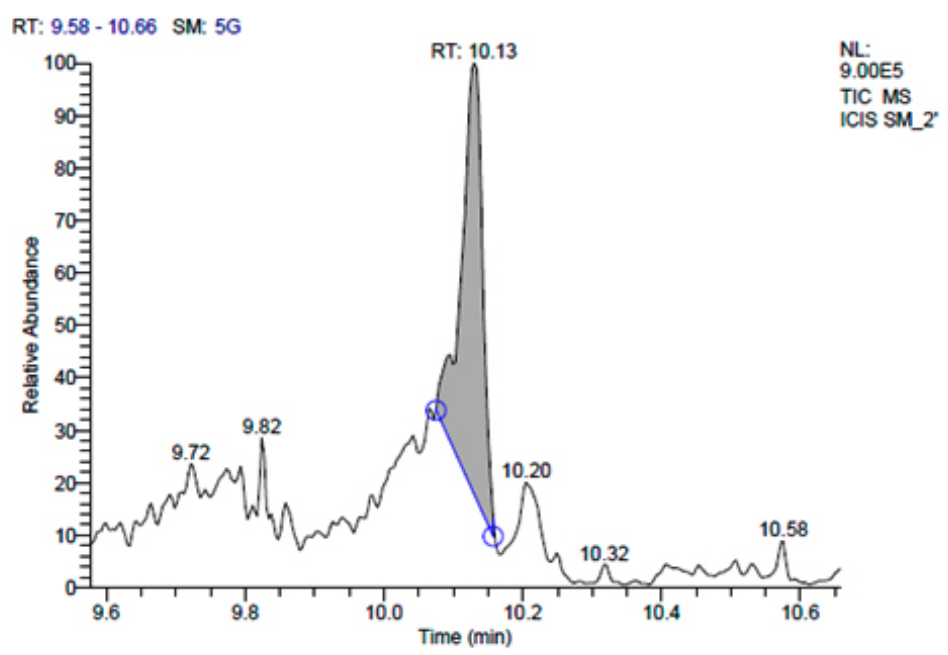

(j) GC-MS chromatogram of $P$. pentosaceus SM-2 at retention time $10.13 \mathrm{~min}$

Fig. 1. GC-MS chromatograms of metabolites secreted by Lactic acid bacteria

studied (Maccaferri et al., 2010, Vitali et al., 2010, Garner et al., 2007).

\section{CONCLUSION}

Utilization of probiotic bacteria and their metabolites in medicines, dietary supplements, pharmaceutical preparation and food products are enhancing due to their potential health benefits such as anticarcinogenic activities, constipation, reducing lactose intolerance, regulation of immune function, allergenic diseases such as food allergy, etc. These attributes of probiotic lactic acid bacteria have been described by the production of different primary and secondary metabolites that exert their biological effects either directly or by modifying the immune system.

\section{ACKNOWLEDGEMENTS}

Authors are thankful to Hon'ble Chairperson JV'n Vidushi Garg \& Hon'ble Founder and Advisor JV'n Dr. Panckaj Garg, Jayoti Vidyapeeth Women's University, Jaipur (Rajasthan) for their kind cooperation, encouragement and providing the facilities of University Innovation Centre and other laboratories.

\section{CONFLICT OF INTEREST}

The authors declare that there is no conflict of interest.

\section{FUNDING}

None.

\section{AUTHORS' CONTRIBUTION}

AC conceived and planned the experiments, performed the analysis and wrote the manuscript. KV and BSS supervised the research project.

\section{DATA AVAILABILITY}

All datasets generated or analyzed during this study are included in the manuscript.

\section{ETHICS STATEMENT}

This research does not contain any studies with human participants or animals performed by any of the authors.

\section{REFERENCES}

1. Alreshidi MM, Dunstan RH, Macdonald MM, Smith ND, Gottfries J, Roberts TK. Metabolomic and proteomic responses of Staphylococcus aureus to prolonged cold stress. J Proteome, 2015; 121: 44-55. https://doi. 
org/10.1016/j.jprot.2015.03.010

2. Aneja K R. Experiments in Microbiology, Plant pathology and Biotechnology. Biochemical activities of microorganisms, 4th edn. New age International Publishers, New Delhi, 2003; pp. 245-275.

3. AOAC. 1995. Official methods of analysis of association of official analytical chemists, 16th edn. Vol I and II. Association of Official Analytical Chemists. Arlington, Virginia, USA.

4. Bharat N, Irshad M, Rizvi M A, Fatma T. Antimicrobial and cytotoxic activities of cyanobacteria. International Journal of Innovative Research in Science, Engineering and Technology, $2013 ;$ 2(9): 4328-4343.

5. Bollag D M and Edelstein S J. Protein concentration determination. In: Bollag D M and Edelstein S J, eds. Protein Methods, Wiley-Liss, 1991 New York.

6. Chaudhary A and Saharan BS. Probiotic properties of Lactobacillus plantarum. Journal of Pure and Applied Microbiology, 2019 ; 13(2): 933-948. https://doi. org/10.22207/JPAM.13.2.30

7. Chen M M, Li A L, Sun M C, Feng Z, Meng X C and Wang Y. Optimization of the quenching method for metabolomics analysis of Lactobacillus bulgaricus. Journal of Zhejiang University-Science B (Biomedicine and Biotechnology), 2014 ; 15(4): 333-342. https://doi. org/10.1631/jzus.B1300149

8. Coucheney E, Daniell T J, Chenu C and Nunan N. Gas chromatographic metabolic profiling: a sensitive tool for functional microbial ecology. Journal of Microbial Methods, 2008 ; 75: 491-500. https://doi. org/10.1016/j.mimet.2008.07.029

9. Da Cruz G, Alonso Buriti A, Batista F C, De Souza C H, Fonseca Faria J A and Isay Saad S M. Probiotic cheese: health benefits, technological and stability aspects. Trends in Food Science and Technology, $2009 ; 20(8)$ : 344-354. https://doi.org/10.1016/j.tifs.2009.05.001

10. de Man J, Rogosa M and Sharpe M. A medium for the cultivation of lactobacilli. Journal of Applied Bacteriology, 1960 ; 3:13-135. https://doi. org/10.1111/j.1365-2672.1960.tb00188.x

11. Del Re B, Sgorbati B, Miglioli M and Palenzona D. Adhesion, autoaggregation and hydrophobicity of 13 strains of Bifidobacterium longum. Letters in Applied Microbiology, 2000 ; 31:438-442. https://doi. org/10.1046/j.1365-2672.2000.00845.x

12. Ezhilan B P and Neelamegam R. GC-MS analysis of phytocomponents in the ethanol extract of Polygonum chinense L. Pharmacognosy Research, $2012 ; 4(1)$ : 11-14. https://doi.org/10.4103/0974-8490.91028

13. Fernandez L, Langa S, Martin V, Maldonado A, Jimenez $E$, Martin R and Rodriguez J M. The human milk microbiota: origin and potential roles in health and diseases. Pharmacological Research, 2013 ; 69(1): 1-10. https://doi.org/10.1016/j.phrs.2012.09.001

14. Garner C E, Smith S, de Lacy Costelllo B, White P, Spencer R. Volatile organic compounds from feces and their potential for diagnosis of gastrointestinal diseases. FASEB J, 2007 ; 21: 1675-88. https://doi. org/10.1096/fj.06-6927com

15. Harrigan W F. Laboratory methods in food microbiology. Academic Press: New York 1998.

16. Harrigan W F and McCance M E. Laboratory Methods in
Food and Dairy Microbiology. Academic Press, London. 1990.

17. Hashem S, Sabit H H, Amin M, Tawakkot W and Shamseldin A F. Molecular characterization of Egyptian isolates of Lactobacillus and Bifidobacterium. Journal of American Science, 2010 ; 6(11): 959-964.

18. Hong-Xin J, Mi-Ya S and Guang-Yu G. Influence of Lactobacillus casei LC2W on the proteolysis and aroma compounds of Cheddar cheese during ripening period. CyTA Journal of Food; 2015 . https://doi.org/10.1080/ 19476337.2014.1003099

19. Lee J, Hong $Y$ and Lee C. Characterization of fermentation behaviours of lactic acid bacteria in Grape wines through $1 \mathrm{H}$ NMR- and GC based metabolic profiling. Journal of Agricultural and Food Chemistry, 2009 ; 57: 4810-4817. https://doi. org/10.1021/jf900502a

20. Li X, Long D, Ji J, Yang W, Zeng Z, Guo S. Sample preparation for the metabolomics investigation of polygamma-glutamate-producing Bacillus licheniformis by GC-MS. J Microbiol Meth, 2013 ; 94(1):61-7. https:// doi.org/10.1016/j.mimet.2013.04.006

21. Liong M T and Shah N P. Acid and bile tolerance and cholesterol removal ability of Lactobacilli strains. Journal of Dairy Science, $2005 ; 88: 55-56$. https://doi. org/10.3168/jds.S0022-0302(05)72662-X

22. Maccaferri S, Vitali B, Klinder A, Kolida S, Ndagijimana M. Rifaximin modulates the colonic microbiota of patients with Crohn's disease: an in vitro approach using a continuous culture colonic model system. J Antimicrobial Chemotherapy, 2010 ; 65: 2556-2565. https://doi.org/10.1093/jac/dkq345

23. Mastrangelo A, Ferrarini A, Rey-Stolle F, Garcia A, Barbas C. From sample treatment to biomarker discovery: a tutorial for untargeted metabolomics based on GC-(EI)Q-MS. Anal Chim Acta, 2015 ; 900:21-35. https://doi. org/10.1016/j.aca.2015.10.001

24. Mora D, Fortina M G, Parini C, Ricci G, Gatti M, Giraffa $G$ and Manachini P L. Genetic diversity and technological properties of Streptococcus thermophilus strains isolated from dairy products. Journal of Applied Microbiology, 2002; 93:278-287. https://doi.org/10.1046/j.1365-2672.2002.01696.x

25. Nordstrom A. Design of experiments: an efficient strategy to identify factors influencing extraction and derivatization of Arabidopsis thaliana samples in metabolomic studies with gas chromatography/mass spectrometry. Anal Biochem. 2004 ; 331(2):283-95. https://doi.org/10.1016/j.ab.2004.04.037

26. Padmavathi A R, Abinaya B and Pandian S K. Phenol, 2,4-bis(1,1-dimethylethyl) of marine bacterial origin inhibits quorum sensing mediated biofilm formation in the uropathogen Serratia marcescens. Biofouling , 2014 ; 30(9): 1111-1122. https://doi.org/10.1080/08 927014.2014.972386

27. Papagianni M and Anastasiadou S. Pediocins: the bacteriocins of Pediococci: sources, production, properties and applications. Microbial Cell Factories, 2009 8:3. https://doi.org/10.1186/1475-2859-8-3

28. Park S E, Seo S H, Kim E J, Byun S H, Na C S, Son H S. Changes of microbial community and metabolite in kimchi inoculated with different microbial community 
starters. Food Chem, 2019; 274: 558-565. https://doi. org/10.1016/j.foodchem.2018.09.032

29. Park S E, Yoo S A, Seo S H, Lee K I, Na C S, Son H S. GC-MS based metabolomics approach of kimchi for the understanding of Lactobacillus plantarum fermentation characteristics. Lwt-food Sci Technol. 2016; 68: 313-321. https://doi.org/10.1016/j. Iwt.2015.12.046

30. Pogacic T, Maillard M B, Leclerc A, Herve C, Chuat V, Yee A L, Valence F, Thierry A. A methodological approach to screen diverse cheese-related bacteria for their ability to produce aroma compounds. Food Microbiol, 2015 46: 145-153. https://doi. org/10.1016/j.fm.2014.07.018

31. Rosenberg M, Gutnick D and Rosenberg E. Adherence of bacteria to hydrocarbons: a simple method for measuring cell surface hydrophobicity. FEMS Microbiology Letters, 1980 ; 9:29-33. https://doi. org/10.1111/j.1574-6968.1980.tb05599.x

32. Schummer C, Delhomme O, Appenzeller B M, Wennig $R$, Millet M. Comparison of MTBSTFA and BSTFA in derivatization reactions of polar compounds prior to GC/MS analysis. Talanta, $2009 ; 77(4): 1473-82$. https://doi.org/10.1016/j.talanta.2008.09.043

33. Settanni L and Moschetti G. Non-starter lactic acid bacteria used to improve quality and provide health benefits. Food Microbiology, 2010 ; 27(6): 691-697. https://doi.org/10.1016/j.fm.2010.05.023

34. Sheela T, Devi D B, Sabetha B, Vinoth M and Jayakumar K. Biocontrol efficacy of synbiotic Strawberry (Fragaria ananassa Duch.) juice. International Journal of food Nutrition and Safety, 2015 ; 6(1): 10-16.

35. Sheela T, Kulothungan $\mathrm{S}$ and Sugnaya R S. Effect of symbiotic milk beverage on diarrhoel causing organisms. International Journal of Current Pharmaceutical Research, $2012 ;$ 4(2): 74-78.

36. Smart K F, Aggio R B M, Van Houtte J R, Villas-Boas
S G. Analytical platform for metabolome analysis of microbial cells using methyl chloroformate derivatization followed by gas chromatography-mass spectrometry. Nat Protoc. 2010 ; 5(10):1709-29. https://doi.org/10.1038/nprot.2010.108

37. Thirabunyanon $\mathrm{M}$, Boonprasom $\mathrm{P}$ and Niamsup $\mathrm{P}$. Probiotic potential of lactic acid bacteria isolated from fermented dairy milks on antiproliferation of colon cancer cells. Biotechnology Letters, 2009 ; 31: 571-576. https://doi.org/10.1007/s10529-008-9902-3

38. Vanaja G, Gotcheva V, Angelov A and Agrawal R. Formation of volatile and fatty acids of therapeutic importance in the probiotic Lactobacillus plantarum LPcfr adapted to resist GI conditions. Journal of Food Science and Technology, 2011; 48(1): 110-113. https:// doi.org/10.1007/s13197-010-0110-5

39. Villas-Boas S G, Mas S, Akesson M, Smedsgaard J, Nielsen J. Mass spectrometry in metabolome analysis. Mass Spectrom. Rev. 2005 ; 24: 613-646. https://doi. org/10.1002/mas.20032

40. Vitali B, Ndagijimana M, Cruciani F, Carnevali P, Candela M. Impact of a synbiotic food on the gut microbial ecology and metabolic profiles. BMC Microbiol, 2010; 10: 4. https://doi.org/10.1186/1471-2180-10-4

41. Vitali B, Ndagijimana M, Maccaferri S, Biagi E, Guerzoni M E and Bridigi P. An in vitro evaluation of the effects of probiotic and prebiotics and prebiotics on the metabolic profile of human microbiota. Anaerobe, 2012; 1: 6. https://doi.org/10.1016/j. anaerobe.2012.04.014

42. Walker D K and Gilliland S E. Relationships among bile tolerance, bile salts deconjugation and assimilation of cholesterol by Lactobacillus acidophilus. Journal of Dairy Science, 1993; 76: 956-961. https://doi. org/10.3168/jds.s0022-0302(93)77422-6 